\title{
Chemical Modification of Colloidal Masks for Nanolithography
}

\author{
Dirk L. J. Vossen, ${ }^{\dagger}$ Joan J. Penninkhof, ${ }^{\dagger,}$ and Alfons van Blaaderen*, ${ }^{\dagger}$ \\ Soft Condensed Matter, Utrecht University, Princetonplein 5, 3584 CC Utrecht, The Netherlands, and \\ FOM Institute for Atomic and Molecular Physics, Center for Nanophotonics, Kruislaan 407, \\ 1098 SJ Amsterdam, The Netherlands
}

Received December 10, 2007. Revised Manuscript Received March 12, 2008

\begin{abstract}
A method is presented to tune the holes in colloidal masks used for nanolithography. Using a simple wet-chemical method, a thin layer of silica is grown on masks of silica particles. The size of the holes is controlled by the amount of tetraethoxysilane (TEOS) added. More accurate tuning of the hole size is possible in the presence of a calibrated seed dispersion of silica colloids. We demonstrate modified masks that were used to create arrays of metal nanoparticles with a size ranging from $400 \mathrm{~nm}$, for unmodified masks, down to tens of nanometers. The method is easy-to-use, fast, and inexpensive.
\end{abstract}

\section{Introduction}

In nanosphere lithography, a self-organized layer of colloidal spheres is used as a mask for a lithographic step like illumination, deposition, or etching. ${ }^{1-5}$ It is a simple, fast, and inexpensive method to pattern substrates over large areas. In this paper, we demonstrate improvements on this technique and use the modified colloidal masks to make arrays of noble metal nanoparticles. These arrays of metal particles have many applications ${ }^{6-8}$ and arrays created using colloidal masks were used, for example, to study plasmonic resonances, ${ }^{9}$ to fabricate plasmonic sensors, ${ }^{10}$ to catalyze the growth of nanofibers, ${ }^{11,12}$ and for selective protein adsorption. ${ }^{13}$

Nanosphere lithography also has disadvantages: one of them is that the size of holes in the mask is determined by the size of the colloidal particles that form the mask. Therefore, the size of the features created using nanosphere lithography is coupled to the size of the colloidal particles in the mask. ${ }^{3,4}$ Recently, we used optical tweezers to create masks with arbitrary geometry, alleviating another limitation of nanosphere lithography, and we used ion beam deformation to control the hole size in colloidal masks. ${ }^{14}$ Control over the hole size was also achieved by deforming colloidal masks made of polystyrene colloids and by evaporation at large off-normal angles in combination with rotation of the sample. ${ }^{15-17}$

\footnotetext{
* Corresponding author. E-mail: a.vanblaaderen@phys.uu.nl.

${ }^{\dagger}$ Utrecht University.

* FOM Institute for Atomic and Molecular Physics.

(1) Fischer, U. C.; Zingsheim, H. P. J. Vac. Sci. Technol. 1981, 19, 881-885.

(2) Deckman, H. W.; Dunsmuir, J. H. Appl. Phys. Lett. 1982, 41, 377-379.

(3) Hulteen, J. C.; Van Duyne, R. P. J. Vac. Sci. Technol. A 1995, 13, 1553-

(4) Burmeister, F.; Schafle, C.; Matthes, T.; Bohmisch, M.; Boneberg, J.; Leiderer, P. Langmuir 1997, 13, 2983-2987.

(5) Yang, S. M; J, S. G; Choi, D. G; Kim, S; Yu, H. K. Small 2006, 2, 458

(6) Shipway, A. N.; Katz, E.; Willner, I. ChemPhysChem 2000, 1, 18-52.

(7) Redl, F. X.; Cho, K. S.; Murray, C. B.; O’Brien, S. Nature 2003, 423, 968-971.

(8) Alivisatos, P. Nat. Biotechnol. 2004, 22, 47-52.

(9) Haynes, C. L.; Van Duyne, R. P. J. Phys. Chem. B 2001, 105, 5599-5611.

(10) Malinsky, M. D.; Kelly, K. L.; Schatz, G. C.; Van Duyne, R. P. J. Am. Chem. Soc. 2001, 123, 1471-1482.

(11) Huang, Z. P.; Carnahan, D. L.; Rybczynski, J.; Giersig, M.; Sennett, M.; Wang, D. Z.; Wen, J. G.; Kempa, K.; Ren, Z. F. Appl. Phys. Lett. 2003, 82, 460-462.

(12) Wang, X.; Summers, C. J.; Wang, Z. L. Nano Lett. 2004, 4, 423-426.

(13) Michel, R.; Reviakine, I.; Sutherland, D.; Fokas, C.; Csucs, G.; Danuser, G.; Spencer, N. D.; Textor, M. Langmuir 2002, 18, 8580-8586.

(14) Vossen, D. L. J.; Fific, D.; Penninkhof, J.; van Dillen, T.; Polman, A.; van Blaaderen, A. Nano Lett. 2005, 5, 1175.
} 1558.
Here we present a simple and fast alternative method to modify the hole sizes of colloidal masks for nanolithography. A wetchemical method is used to grow a thin layer of silica on twodimensional close-packed layers of colloidal silica particles on a substrate. The layer of silica decreases the size of the holes in the colloidal mask and the hole size is uncoupled from the interhole distances; it is now, for example, possible to obtain small nanoparticles with large interparticle distances. We investigated the growth mechanism of the silica coating and show that the size of the holes can be controlled accurately. The modified masks were used for nanolithography: after metal deposition and removal of the mask, large arrays of metal nanoparticles were created. Using masks consisting of large colloidal particles with a diameter of $1.4 \mu \mathrm{m}$, we created nanoparticles with a size ranging from $400 \mathrm{~nm}$ (for unmodified masks) down to tens of nanometers.

The coating method is based on the Stöber method, ${ }^{18}$ a basecatalyzed reaction of alkoxy silanes in mixtures of ethanol and ammonia, which is well-known for the synthesis of monodisperse colloidal silica particles. Catalyzed by the ammonia, tetraethoxysilane (TEOS) molecules hydrolyze and condense to form silica. In the early stage of the reaction, small silica aggregates are formed. These coalesce until they become stable, as they grow larger and acquire more surface charge. Once stable, the aggregates grow larger by addition of monomers and small oligomers and become spherical in shape. The final size of the colloidal spheres depends on both the number of particles formed in the early nucleation stage as well as on the amount of TEOS added to the reaction mixture. The number of particles formed in the stable dispersion is a complicated function of experimental conditions and is in general not accurately controllable. ${ }^{19-21}$

Recently, we developed a modification of the Stöber method to grow thin silica films on macroscopic surfaces. ${ }^{22}$ The growth mechanism for the silica layers was found to be surface-reaction

(15) Kosiorek, A.; Kandulski, W.; Chudzinski, P.; Kempa, K.; Giersig, M. Nano Lett. 2004, 4, 1359-1363.

(16) Kosiorek, A.; Kandulski, W.; Glaczynska, H.; Giersig, M. Small 2005, $1,439$.

(17) Miguez, H.; Tétreault, N.; Hatton, B.; Yang, S. M.; Perovic, D.; Ozin, G. A. Chem. Commun 2002, 2736-2737.

(18) Stöber, W.; Fink, A.; Bohn, E. J. J. Colloid Interface Sci. 1968, 26, 62-69.

(19) Giesche, H. J. Eur. Ceram. Soc. 1994, 14, 205-214.

(20) van Blaaderen, A.; Vrij, A. Langmuir 1992, 8, 2921-2931.

(21) van Blaaderen, A.; van Geest, J.; Vrij, A. J. Colloid Interface Sci. 1992, 154, 481-501. 


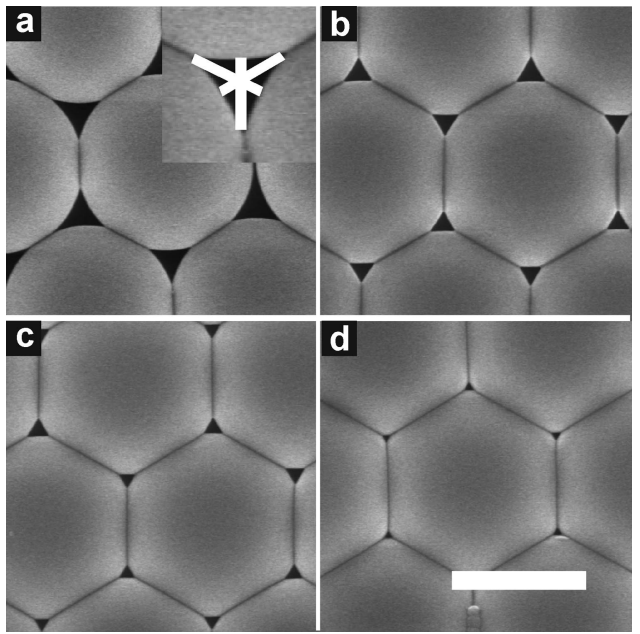

Figure 1. Colloidal masks formed by a layer of close-packed particles (700 $\mathrm{nm}$ radius), modified by growing thin layers of silica to tune the hole size. The size of the holes in an uncoated mask was $400 \mathrm{~nm}$. (a) SEM image of a mask after one coating step; the size of the holes reduced to $264 \mathrm{~nm}$. The inset indicates the definition of hole size. $(\mathrm{b}-\mathrm{d})$ Images of colloidal masks after two, three, and four coating steps. The size of the holes was 152, 104, and $55 \mathrm{~nm}$, respectively. The scale bar is $1 \mu \mathrm{m}$.

limited, as was also found for the original Stöber process. ${ }^{21}$ It is therefore expected that when a seed dispersion of colloidal spheres is added to the reaction mixture, the thickness of the silica layer on a macroscopic surface increases with the same amount as the increase in radius of the colloidal seed particles. This was indeed what was found. ${ }^{22}$ When a well-characterized seed dispersion is used, silica layers can be grown with nanometer control over layer thickness. ${ }^{22}$ We use this method to grow thin silica layers on colloidal masks for nanolithography.

\section{Experimental Section}

Silica particles with a diameter of $1.4 \mu \mathrm{m}$ and a polydispersity of $2 \%$ were synthesized using a Stöber growth process and subsequent growth steps using methods described in detail elsewhere. ${ }^{19-21}$ The size and polydispersity were determined using scanning electron microscopy (SEM) on several hundreds of particles. Analytical grade ethanol, ammonia solution ( $29 \mathrm{wt} \%$ ) and TEOS from Merck were used as received. $\mathrm{Si}(100)$ wafers were used as substrates, as received.

Hexagonal close-packed masks were made by self-organization of colloidal particles on an uncoated substrate. A drop of particles in ethanol was put on a glass or silicon substrate and the solvent was left to evaporate slowly. The average size of the single crystalline regions was on the order of several tens of particles diameters.

The substrates were coated in a mixture of ethanol, ammonia, water, and TEOS as described in the text. A layer of gold was deposited on the colloidal masks using electron beam evaporation at a base pressure of $1 \times 10^{-8}$ mbar. After deposition, the mask was removed from the sample by sonication (Branson Ultrasonic Cleaner, model $8510 \mathrm{E}$ ) in ethanol. Sometimes, masks coated with a thick silica layer were found to be hard to detach from the substrate.

Scanning electron microscopy (SEM) was performed on a FEI XL30 SFEG microscope operated at an acceleration voltage in the range of $1-15 \mathrm{keV}$. Holes in colloidal masks were characterized by measuring the three long axes of the hole's triangular shape as described in the text and indicated in the inset of Figure 1a. An average over five holes was taken.

\section{Results and Discussion}

A colloidal seed dispersion was synthesized by adding 0.360 $\mathrm{g}$ of TEOS to a mixture of $7.867 \mathrm{~g}$ of ethanol, $0.787 \mathrm{~g}$ of water,

(22) Vossen, D. L. J.; de Dood, M. J. A.; van Dillen, T.; Zijlstra, T.; van der Drift, E.; Polman, A.; van Blaaderen, A. Adv. Mater. 2000, 12, 1434-1437. and $0.715 \mathrm{~g}$ of ammonia. After $2 \mathrm{~h}$ of continuous stirring, colloidal silica particles had formed, and $75 \mu \mathrm{L}$ of seed dispersion was taken out of the reaction mixture. The radius of the newly formed spheres in the seed dispersion was determined to be $363 \mathrm{~nm} \pm$ $2.3 \%$. From this, the number density of seed particles in the dispersion was calculated to be $2.7 \times 10^{11} \mathrm{~mL}^{-1}$.

Four samples with colloidal masks were prepared as described in the Experimental Section. The size of the holes between the particles in the colloidal mask was measured to be $400 \mathrm{~nm}$. The size of a hole is defined as the smallest distance between the point where two particles touch and a point on the surface of the third colloidal particle in a hexagonal close-packed mask (see the inset in Figure 1a). The four substrates covered with colloidal masks were then immersed in the ethanol-ammonia mixture containing the seed dispersion. Subsequently, $0.188 \mathrm{~g}$ of TEOS was added, and after $2 \mathrm{~h}$ of stirring, the reaction was finished, and one substrate and $75 \mu \mathrm{L}$ of seed dispersion were taken out of the mixture. Then $0.178 \mathrm{~g}$ of TEOS was added to the mixture with seed dispersion and the three remaining samples. After $2 \mathrm{~h}$, again one sample and $75 \mu \mathrm{L}$ of seed dispersion were taken out. These steps were repeated twice in which 0.083 and $0.084 \mathrm{~g}$ of TEOS were added, respectively.

The substrates can also be coated without any seed particles present in the reaction mixture, but a secondary nucleation of particles will prevent knowing exactly how thick the layer grown on the substrates will be. By adding seed particles to the dispersion, accurate control over the final thickness of the silica layer is available, as no new particles are formed and the total known surface area dictates also the thickness grown on the template.

Figure 1a shows a SEM image at high magnification of a colloidal mask that was taken out of the reaction mixture after the first coating step. A layer of silica had grown on the mask, and the size of the holes was decreased to $264 \mathrm{~nm}$. This is a 136 $\mathrm{nm}$ reduction of the hole size compared to an uncoated mask. After the second coating, the size of the holes was reduced to $152 \mathrm{~nm}$ (Figure 1b). After the third and fourth coating steps, the size of the holes were determined to be $104 \mathrm{~nm}$ (Figure 1c) and $55 \mathrm{~nm}$ (Figure 1d), respectively. Virtually no seed particles were found on the coated colloidal masks.

During each coating step, silica was grown on the colloidal masks and on the seed colloids in the reaction mixture. We determined the size of the seed and mask particles after each growth step by electron microscopy. The size of the mask particles is defined as the radius of a particle in the mask at the position where it is not connected to another particle. The graph in Figure 2 shows the increase of the radius of both the seed and mask particles as a function of the amount of TEOS added. The size of the holes in the mask after each growth step is also indicated in the graph. With more TEOS added, the size of the seed particles increased more rapidly than the size of the particles in the mask.

The Stöber growth of silica colloids is well-described by a surface reaction-limited model, in which the growth rate is limited by condensation of hydrolyzed monomers on the particle surface. ${ }^{21,23}$ The change in particle volume is then proportional to the square of the particle radius, and the increase in particle radius will be independent of size. In contrast, the growth rate in diffusion-limited growth is limited by the transport of monomers to the particle surface, and the growth in particle volume is then proportional to the radius of the particles. The observed difference in growth rate for seed and mask particles in Figure 2 indicates that the silica growth on mask particles is more diffusion-limited than reaction-limited. Although diffusion-limited growth means

(23) Chen, S.-L.; Dong, P.; Guang-Hua, Y. J. Colloid Interface Sci. 1997, 189, 268-272. 


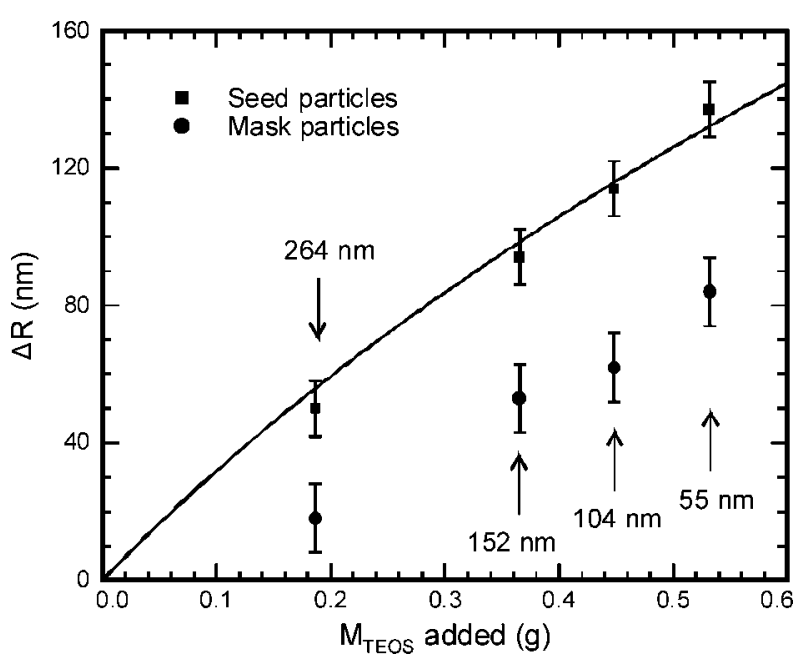

Figure 2. The size increase of the seed particles in the coating mixture (squares) and the colloidal particles in the mask (circles) as a function of amount of TEOS added. The size of both particles increased, although the seed particles grew faster. For each coating step, the hole size in the mask is also indicated. The curve shows the expected relation for the surface reaction-limited growth model.

that the layer thickness grown on the colloidal mask will depend on geometrical properties of the mask, which will be different for different samples, accurate tuning of the size of the holes is still possible when a calibration curve is determined.

For seeded growth, it was shown that the amount of TEOS that has to be added to increase the radius $R_{1}$ to $R_{2}$ is related to the volume of TEOS $\left(V_{1}\right)$ needed to prepare the particles with radius $R_{1}{ }^{21}$

Here, it is assumed that all the added TEOS grows on the existing particles and that the density of the added layer is the same as that of the core particle. This formula can be applied to the growth of the seed colloids, as the surface provided by the substrates with the masks is negligible. The result, with $R_{1}=374$ $\mathrm{nm}$ as determined by SEM, is shown by the solid line in Figure 2 , and shows a good agreement with the data.

After modification, the masks were used for lithography. A layer of $25 \mathrm{~nm}$ of Au was deposited on a modified colloidal mask by electron beam evaporation. After removal of the mask by sonication, an array of Au nanoparticles was left on the sample (Figure 3a). The size of the nanoparticles was determined to be $70 \mathrm{~nm}$. Since the substrate was also coated during the coating, the metal nanoparticles were deposited on a thin layer of silica. Where the colloidal mask touched the silicon substrate, indentations in the silica film can be seen.

To investigate the height profile of a sample, an array of $\mathrm{Au}$ particles was imaged using an atomic force microscope (AFM) (Figure 3b). The Au particles had a lateral size of $350 \mathrm{~nm}$. Height profiles recorded on the two dashed lines indicated in Figure $3 \mathrm{~b}$ are plotted in Figure 3c. Each line shows two Au particles on the silica layer on top of the silicon substrate. On average, the height of the Au particles was $27 \mathrm{~nm}$, while the silica layer had a thickness of $29 \mathrm{~nm}$. For many applications, the presence of silica on the substrate poses no problem. However, modification
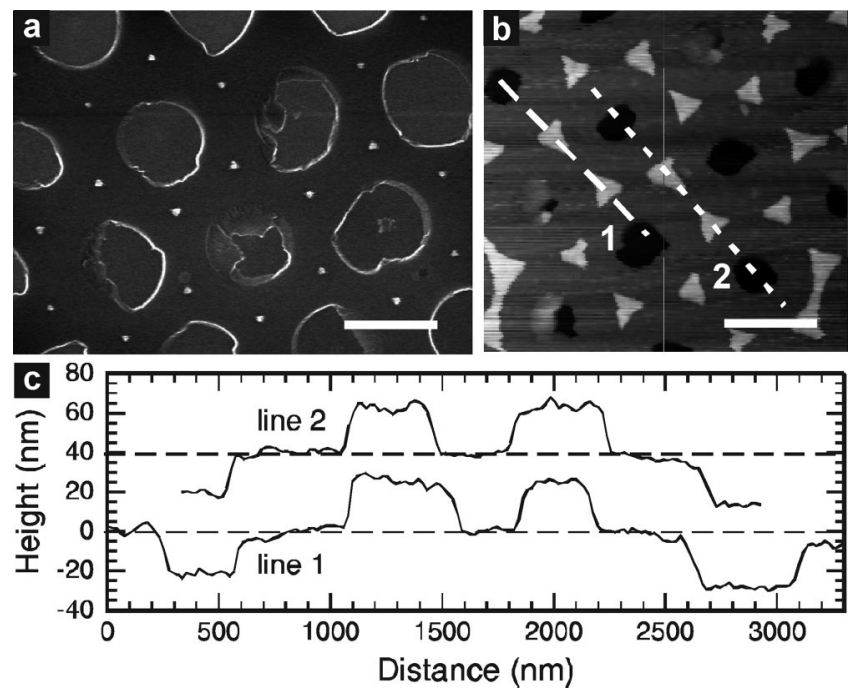

Figure 3. (a) SEM image of an array of gold nanoparticles created by deposition of $25 \mathrm{~nm}$ of Au on a modified colloidal mask and subsequent mask removal by sonication of the sample. The in-plane size of the nanoparticles was $70 \mathrm{~nm}$. (b) AFM image of an array of Au particles $(350 \mathrm{~nm})$ on a silica layer. The holes in the silica layer are caused by the particles in the mask during the coating. (c) Height profiles over two $\mathrm{Au}$ particles on the silica layer on the silicon substrate. The height of the Au particles was $27 \mathrm{~nm}$ while the silica layer had a thickness of 29 $\mathrm{nm}$. The positions where the profiles were taken are indicated in Figure $3 \mathrm{~b}$ with the dashed lines. The scale bars are $1 \mu \mathrm{m}$.

of the surface can be prevented when the method is used on freestanding colloidal masks that are then transferred to a substrate for lithography. ${ }^{4}$ We have shown previously that silica can be grown on many different materials (including latex spheres) using the polymer poly(vinylpyrrolidone), so that this modification method can be applied to a variety of substrates and colloidal masks. ${ }^{24}$

\section{Conclusions}

In conclusion, we developed a wet-chemical method to modify the hole size of colloidal masks used for lithography. A mask is coated with a thin silica layer and the size of the holes is controlled by the amount of TEOS added. Accurate tuning of the hole size is possible in the presence of a (calibrated) seed dispersion of silica colloids. As an example, the modified masks were used to create arrays of metal nanoparticles. The method is inexpensive, simple, and fast.

Acknowledgment. We thank Damir Fific for experimental assistance, Anna Tchebotareva for AFM imaging, and Albert Polman for discussions. This work is part of the research program of the "Stichting voor Fundamenteel Onderzoek der Materie (FOM)", which is financially supported by the "Nederlandse organisatie voor Wetenschappelijk Onderzoek (NWO)".

\section{LA703847P}

(24) Graf, C.; Vossen, D. L. J.; Imhof, A.; van Blaaderen, A. Langmuir 2003, 19, 6693-6700. 